\title{
The use of experts and their judgments in nursing research: an overview
}

\author{
JC Bruce, PhD \\ Associate Professor, Department of Nursing Education, Faculty of Health Sciences, University of the \\ Witwatersrand, Johannesburg
}

\section{GC Langley, PhD}

Senior Lecturer, Department of Nursing Education, Faculty of Health Sciences, University of the Witwatersrand, Johannesburg

\author{
AA Tjale, PhD \\ Lecturer, Department of Nursing Education, Faculty of Health Sciences, University of the Witwatersrand, \\ Johannesburg
}

Key words:

expert, judgment, judgmental data, nursing research

\section{Correspondence address:}

Professor Judith Bruce

Department of Nursing Education

Faculty of Health Sciences

University of the Witwatersrand

7 York Road

Parktown

2193

Tel: (011) 488-4272/4196

Fax: (011) 488-4195

Email: Judith.bruce@wits.ac.za

\section{Abstract: Curationis 31(4): 57-61}

Experts and their judgments are widely used in the fields of research, education, health care, law, commerce and technology. Expert judgment is known for its subjectivity and its potential for bias, which brings into question the accuracy and authenticity of judgmental data. At the same time there is acknowledgment of the valued contribution of judgmental data towards valid inferences in research and education. Maximizing the use of experts and their judgments has therefore become an endeavour of educationists and researchers alike.

Since this is not a research article its purpose is to guide and assist nurse researchers with important methodological and ethical decisions when using experts. Experts must be used in the context of appropriate research methods such as the Delphi and Nominal Group techniques. Sampling of experts and sample size is determined by the type and quality of data and the availability of population data: purposive and maximum variation sampling techniques are recommended as appropriate when sampling experts. Universal research ethics must be applied with particular consideration of aspects which may influence the truth value of consensus among experts and marginalization of minority or extreme viewpoints. Quantification of judgmental data is recommended and is important to minimize bias and to increase the authenticity of research findings.

The content includes: design considerations when using experts, sampling issues, ethical rules to be considered when enlisting experts and their judgments, optimal data collection approaches and managing judgmental data. 


\section{Introduction}

The use of expert judgments has become increasingly popular in the fields of research, education, health care, law, commerce and technology. Experts are defined as informed persons in a particular discipline or field, usually constituted as a panel, as individual members or both (Burns \& Grove, $2005: 400$ ). In this case the field is nursing research, defined as systematic enquiry designed to develop knowledge in the nursing profession (Polit \& Beck, 2004:726). Experts are used mainly, but not exclusively, for the purpose of judging the content-related evidence of evaluation instruments and data collection schedules in education and research. Judging refers to experts giving their professional opinion about desirable outcomes (Gomm, Needham \& Bullman, 2000:81), During this (judging) process experts are asked how well each of the concepts or items has been represented in the instrument (Burns \& Grove, 2005: 401) or the relative value (weight) of items.

Expert judgment is known for its subjectivity and its potential for bias, which brings into the question the accuracy and authenticity of judgmental data. At the same time there is acknowledgment of the valued contribution of judgmental data towards valid inferences in research and education. Maximizing the use of experts and their judgments has therefore become an endeavour of educationists and researchers alike. This paper discusses the best measures for selecting experts, for designing and implementing methods to collect and manage judgmental data and outlines the ethical considerations when using experts in research. Since this is not a research article its purpose is to guide and assist nurse researchers with important methodological and ethical decisions when using experts.

\section{Literature Review}

Judgment by experts has been used in research studies in a range of diverse fields. Apart from nursing other fields of use include medical education (Downing, Tekian, \& Yudkowsky, 2006:51), the military (Crawford \& Williams, 1985:387) and in psychology and social science research (Miranda, 2001:87). In most of these fields problems have surfaced in both acquiring and managing judgmental data (Crawford \& Williams, 1985:388). Additionally, there is mounting criticism that expert judgments are less transparent, biased towards overestimating the value of an item/ concept and naturally so, subjective. In this regard authors (Crawford \& Williams, 1985:387; Miranda, 2001:88) refer to the term "subjective judgment". As a result of these problems and criticisms researchers have made considerable strides towards the quantitative analysis of subjective data to minimize inherent biases and to improve its accuracy. However, deciding when to use experts and who these experts would be are important precursors to generating judgmental data from experts.

\section{Who is an "expert"?}

Apart from being described as being knowledgeable or an informed person in a particular field or discipline there are no specific directives in the literature about the defining characteristics of an expert. The decision about who qualifies to be an expert and who will be selected as such, lies mainly with the researcher. It is therefore important that the researcher examines his/her own values and assumptions about what knowledge is, how it is acquired and developed, and what then does it mean to be "knowledgeable". Benner (1984:2) refers to the work of philosophers of science such as Kuhn and Polanyi in describing "knowing how" and "knowing that" and posits that many of our skills (knowing how) are learnt without scientific formulations of "knowing that".

In a practice discipline such as nursing and as a consequence in nursing research, knowledge embedded in practice becomes important for deciding who is an expert. If one uses Benner's (1984) seminal work "From novice to expert", expertise develops when someone tests and refines propositions, hypotheses and principles in actual practice situations; an expert then no longer relies on rules/principles for judgment or decision-making.

Experience is therefore a requisite for expertise (Benner, 1984: 3); the richness of experience leads an expert to have intuitive knowledge that arises from a deep understanding of the total situation (Benner, 1984: 32). Decisions around experience would thus be informed by how active the person is in the field and the nature of activity, or what the breadth/ scope of the experience is. Deciding who would qualify as an expert also depends on the purpose the data should serve.

\section{When to use experts}

Experts have been used for various purposes in a range of fields. In research and education experts have been used consistently for the design, development and testing of instruments to collect data. In particular, expert judgments have been used and quantified to determine content and construct validity and to assign component weights to assessment items that are assumed to be of equal importance or weighting.

Through consensus building, experts may also be used to develop research agendas for determining research priorities in a particular field (Nathens, Cook \& Machiedo, 2006:101). Developing research agendas in this way is particularly useful in research environments challenged by lack of funding or where funds are best spent on addressing research questions already decided upon by experts and have been ranked through expert consensus.

Experts are also useful for idea creation to improve on the quality of ideas generated and for idea building (Benjamin, Archibold \& Suarez, 2004:1). In idea building it is the interaction between experts, constituted as a panel, that adds richness to the process and hence the data generated. Selecting some experts for their subject expertise and others for their creativity is an important sampling consideration (Benjamin et al., 2004:2).

In health care, business and economics experts are increasingly being called upon to evaluate new technologies (Benjamin et al., 2004:2) and policies and to recommend actions on matters of importance. Panels of experts and individual experts have been found useful when historical data are limited or where ethical and moral issues need to be teased out in a broad or complex problem (Yousuf, 2007:6). In management practice, experts play an important role in evaluation and selection processes and to provide future directions in planning.

\section{Design and methods when using experts}

Within both quantitative and qualitative 
approaches there are several research designs and methods that require the use of experts as a data source. The most commonly proposed methods are the Delphi technique, the Nominal Group Technique and surveys (Burns \& Grove, 2005:436; Polit \& Beck, 2004:238; Steward, 2001:299; Yousuf, 2007:2). Surveys are used in the broadest sense to describe the overall design of a study using experts and not as a specific method to collect judgmental data.

The Delphi technique is essentially a group process whereby the opinions and judgments of a group of identified experts are elicited on a specified topic usually through a series of questions (Yousuf, 2007:3). It may be used to gain expert consensus, called normative Delphi and to establish norms and standards by giving structure to a set of properties (Yousuf, 2007:3). This is in contrast to a policy or decision Delphi, which seeks to elicit divergent responses from experts.

The Nominal Group Technique (NGT) is similar to Delphi, except that the NGT requires the physical presence of members in the form of an expert panel. The researcher would normally act as facilitator of the group process. The NGT is primarily a consensus building tool that assists to rank or prioritize issues or items. When deciding to use experts and their judgments the researcher must be clear about the purpose the data should serve. Knowing the purpose guides the researcher to selecting the sample, choosing an appropriate data collection method and hence, data management strategies.

\section{Sampling considerations}

Sampling of experts must be guided by the type and quality of judgmental data to be obtained. Purposive sampling, also known as judgmental sampling, has been widely supported as an appropriate method of sample selection especially in qualitative research and sometimes in quantitative research (Burns \& Grove, 2007: 331). Purposive sampling is broadly defined as "Selecting participants based on personal judgment about which ones will be more representative or informative..." (Polit \& Beck, 2004:729). However, it is difficult to evaluate the accuracy of the researcher's judgment (Burns \& Grove, 2007:345); purposive sampling therefore continues to be criticized for the lack of methods in support of the representativeness or typicalness of the sample selected. Purposeful selection requires the identification of experts in disciplines or domains directly and indirectly represented in the research instrument or topic under discussion, and who could provide rich data as evidence for the content-related validity of the instrument. Ideally, these disciplines or domains must also be sampled to ensure richness of judgmental data. Nesting purposive sampling within maximum variation sampling is one way to obtain representativeness and rich data by including a wide range of extremes (List, 2004: 1; Patton, 2002:234).

Maximum variation or heterogeneity sampling is described as a special kind of purposive sampling (List, 2004:2), which may be used to identify experts or cases (in qualitative research) to provide rich information (Patton, 2002:234). This sampling method aims to identify themes or patterns that run through a range of variations (Patton, 2002:234); it is informed by the logic that any common themes that emerge from great variation are valuable in capturing the core experiences of a setting or phenomenon. In quantitative terms this type of sampling is also an extension of the statistical principle of regression towards the mean, which means that in a group of experts who is extreme in several different ways one is likely to find some who are average in other ways (List, 2004:2). Thus maximum variation sampling limits the possibility of selecting a narrow sample (few cases) from one with wide variation. According to Yousuf (2007:4) heterogeneity of participants must be preserved to arrive at valid results. When experts are similar they are more likely to agree than to disagree resulting in artificial consensus. In a group or panel situation domination by number or by personality may lead to the "bandwagon effect" (Linstone \& Turoff, 1975:4). When to use maximum variation sampling depends on the availability of population data and on sample size.

\section{Sample size considerations}

Sample size is determined by the study design (Yousuf, 2007:6) and the aims and purpose of the experts' undertaking, which in turn informs the sampling method. If consensus among experts is the aim for example in validation studies a sample of between 5-10 experts is recommended (Burns \& Grove, 2005: 400). In areas where expertise is limited a minimum of three experts is recommended, which will require $100 \%$ agreement between them if a consensus Delphi is used. The lower the number of experts the greater the demand for $100 \%$ agreement; more experts allows for greater variation in their level of agreement and hence, their judgment. The level of agreement must be set by the researcher prior to implementation; generally $75-80 \%$ is the lowest acceptable level of agreement.

Maximum variation sampling is appropriate for consensus groups of approximately three within samples of 15 20 and for samples of 20-50 for surveys of expert opinions (List, 2004:2). This type of sampling is particularly useful if the target population from which experts may be drawn is not clearly defined or there a great variation in the domain or phenomenon to be studied. Maximum variation sampling requires identification of extremes in a particular context followed by purposive selection of participants. Examples include: variations in workplace settings of nurses (Cheek \& Jones, 2003:42), variations in qualifications, work experience, expertise and practice domain of academics in universities, and extremes in age, level of leisure and work activities in a village community (List, 2004:3) when conducting research in communities.

\section{Ways to collect data from experts}

After identifying and selecting the experts the study design decided upon is followed to collect data from experts' judgments. To ensure good data quality it is important firstly, to provide necessary information about the process and give clear instructions to experts usually by way of an information letter or a meeting with them. Secondly and if applicable, decide on and include the scale to capture judgmental data for example: Likert scale, Visual Analogue Scale, Graphs; tracings and video recordings; this includes clear criteria or directives on how to apply the scale. Thirdly, if consensus is required decide on the level of agreement between experts. Lastly, build in suitable time frames according to the study design and practicality, and to get commitment from experts. Within 
Figure 1 - Example of use of a Visual Analogue Scale

Verbal expression (A) vs. Non-verbal cues (B)

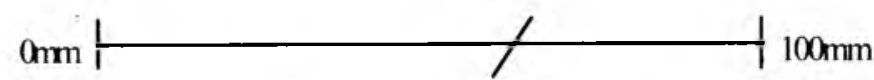

A

B

the Delphi technique about 30 to 45 days are required to complete data collection (Yousuf, 2007:5).

\section{Managing judgmental data}

Quantifying judgmental data is a process that has become increasingly important to make optimal use of experts' judgments. To begin this process the researcher must choose a suitable data management system e.g. Excel spread sheet to prepare judgmental data for analysis. Some procedures for quantifying judgmental data sets are described below. These include: calculating the Content Validity Index (Polit \& Beck, 2004:423) and correlation coefficients, applying the Judgmental Policy Capturing procedure (Harvill, Lang \& McCord, 2004:1) and the Subjective Judgment Model (Crawford \& Williams, 1985:387) to experts' judgments.

The quality of measuring a particular construct is dependent on the degree to which the measurement method or instrument includes all the relevant elements of that construct (Burns \& Grove, 2005:400); that means determining the content-related validity for example, through the use of expert judgment. Although there are several sources of evidence for content-related validity, calculating the content validity index (CVI) is a way to quantify experts' judgments. Using a 4-point Likert scale (Polit \& Beck, 2004:423) experts rate the content relevance of each item and/or sub-item. Judgment quantification involves firstly, determining the proportion of experts who must agree for content validity to be established and secondly, calculating the CVI. The actual CVI is the proportion of items that received a rating of 3 or 4 by the experts; a CVI of .80 or more indicates high content validity (Polit \& Beck, 2004:423).

For consensus data sets such as the above, Pearson's correlation coefficient (a statistic that expresses the size and direction of an association between two variables) may be used to assess statistical relations between variables. For example between the mean score of each item/question and the percentage of experts who scored an item as important/relevant or very important/ very relevant, that is, at the higher end of the scale (Nathens et al., 2006:107). A high correlation coefficient (closer to +1 or -1 ) is desirable and rules out, to a large extent, the possibility of chance agreement. In ranked or prioritized items, increasing mean scores and decreasing variability in scoring within each item, as expressed by the Standard Deviation (SD), usually suggest a high degree of consensus for highest ranked items.

Judgmental Policy Capturing (JPC) is a procedure to statistically describe the information processing strategies of experts (Harvill et al., 2004:1). During this process experts are required to review, independently, the components of a complex, multidimensional assessment (not for individual OSCE procedures or "test items") and decide which of the components are more important than others (Harvill et al., 2004:2). By applying multiple regression analyses, appropriate weights are determined for components in an assessment.

Subjective Judgment Model (SJM) enables subjective, pair-wise comparison of items by experts whose judgments are captured on visual analogue scales (100mm in length) or a judgment matrix. Usually data from visual analogue scales are based on an expert's educated guess on one variable; using the SJM requires the expert to judge the importance of one variable in relation to another, for example verbal expression vs. non-verbal cues (Figure 1). Mathematical procedures are applied to determine the relative weights of the variables or items. In contrast to the above (JPC) this method is useful for less complex assessments or individual instruments.

\section{Ethical considerations when using experts}

When experts are invited to participate in research, their informed consent, written or verbal, should be sought. To be truly informed, all relevant facts must be disclosed and understood by the potential participant and a decision made to voluntarily contribute to the study arrived at without undue coercion. All relevant facts include the purpose, risks, benefits and social implications of participation. The latter is of particular importance. Where the results of the research conflict with established authority or policy and the distinctive influence of the expert can be detected, or his or her identity surmised, then the principles of confidentiality and anonymity might be jeopardized and the person's good name might be put at risk. This is especially relevant where expertise is limited and only one or two experts are able to be accessed or where experts are well known in a discipline or geographical location.

Where participation will involve membership in a group, the invitee has the right to know of the other members of the group. This is to ensure privacy the control by the individual over the extent, timing and circumstances of sharing oneself with others (Rischbieth \& Blythe, 2005:49). The right to refuse participation, to decline to answer questions posed or to withdraw at any stage of the process without any penalty or consequence must be assured prior to eliciting participation.

It is important to ensure confidentiality of data containing opinions, survey results or interviews, which experts will be requested to comment on. All information which may lead to the identification of primary subjects and sites or information, which might pose a threat to personal, occupational, legal or ethical rights, must be removed. This is of particular import where the topic under investigation could be considered illegal, immoral or controversial, for example termination of pregnancy, prostitution, child abuse and illegal immigration. Where the data for expert opinion consist of instruments or scales, which have been 
modified or adapted, the experts must be aware that the original holders of the copyright - author/s or institutions have given permission for their property to be used.

The concomitant principle of anonymity is germane here. The expert informant must be aware that, having earned the appellation "expert", it is possible that their identity might be presumed, particularly if expertise in the area is limited or when their views are well known, controversial or contradict the received view. In the case of Nominal Group Technique or a group interaction Delphi the originator of an opinion, comments or rating must not be identifiable. This may be achieved by summarizing anonymously the experts' opinions and justification given for these statements. If the names of the experts, as individuals or as groups, are to be included in the final report, then their consent must be given (Colton \& Hatchet, 2004:2).

Using experts for consensus building raises several ethical concerns. Consensus reached may not be true consensus and may be the result of misleading or manipulated consensus (Yousuf, 2007:4). This leads to data that do not contain best judgments but rather a compromise position. Trustworthiness may be jeopardized when compromise is reached or consensus is manipulated or subtly coerced by powerful or persuasive group members. Not exploring disagreement or marginalizing dissenting voices may also generate artificial consensus. Using expert groups to achieve consensus on controversial topics or morally divisive topics is generally deemed unethical. However, the broad and immediate context from which data are collected must be born in mind; what is contentious, irreligious or morally divisive in one context might not be so in another.

\section{Conclusion}

Inclusion of measures to enhance objectivity of experts' judgments and hence the authenticity of judgmental data has become mandatory in all scientific research. This implies that researchers must have sound reasons for using experts and follow scientific procedures to make optimal use of expert data. Deciding who is an expert requires careful examination of the values and assumptions underpinning the researcher's conceptualizations of knowledge and the acquisition of knowledge. Universal ethical principles must be observed with particular reference to ethical complexities associated with privacy, confidentiality, anonymity and the nature of consensusbuilding. The information obtained from a research study is only as good as the experts who participate in it, and the study design followed; let not poor design and methods adversely influence the quality of experts and their judgments.

\section{References}

BENNER, P 1984: From Novice to Expert: Excellence and Power in Clinical Nursing Practice. Addison-Wesley: California.

BENJAMIN, CO; ARCHIBOLD, E \& SUAREZ, T 2004: Using expert panels to evaluate new technology. Journal of the Academy of Business and Technology. http://findarticles.com/p/ articles/mi_m0OGT/is (Accessed 7 March 2007).

BURNS, N \& GROVE, SK 2005: The Practice of Nursing Research Conduct, Critique and Utilization. W B Saunders Co: New York.

BURNS, N \& GROVE, SK 2007: Understanding Nursing Research, building an evidence-based practice. Saunders Elsevier: St Louis.

CHEEK, J \& JONES, J 2003: What nurses say they do and need: implications for the educational preparation of nurses. Nurse Education Today. 23:40-50.

COLTON, S \& HATCHET, T 2004: The development of a research instrument to analyze the application of adult learning principles to online learning. http:// eric.ed.gov/ERICebPortal/Home

(Accessed 7 March 2007).

CRAWFORD, G \& WILLIAMS, C 1985: A note on the analysis of Subjective Judgment Matrices. Journal of Mathematical Psychology. 29, 387-405.

DOWNING, SM; TEKIAN, A \& YUDKOWSKY, R 2006: Procedures for Establishing Defensible Absolute Passing Scores on Performance Examinations in Health Professions Education. Teaching and Learning in Medicine. 18(1):50-57.
GOMM,R;NEEDHAM,G\& BULLMAN,

A 2000: Evaluating Research in Health and Social Care. SAGE Publications: London.

HARVILL,LM; LANG, FF \& McCORI), RS 2004: Determining Component Weights in a Communications Assessment using Judgmental Policy Capturing. Medical Education online. 9(12):1-8.

LINSTONE, HA \& TUROFF, M 1975:

Philosophical and methodological foundations of Delphi. Addison-Wesley Publishing Company: Massachusetts.

LIST, D 2004: Maximum variation sampling for surveys and consensus groups. Adelaide: Audience Dialogue. Available at www.audiencedialogue.org/ maxvar.html (Accessed 3 July 2007).

MIRANDA, E 2001: Improving Subjective Estimates using Paired Comparisons. IEEE Software. Ericsson Research Canada. January/February 2001,87-91.

NATHENS, AB; COOK, CH, MACHIEDO, G 2006: Defining the Research Agenda for Surgical Infection: A Consensus of Experts using the Delphi Approach. Surgical Infections. 7(2):101110.

PATTON, MQ 2002: Qualitative Research and Evaluation Methods. Sage: Thousand Oaks, California

POLIT, DF \& BECK, CT 2004: Nursing Research Principles and Methods. Lippincott Williams \& Wilkins: Philadelphia.

RISCHBIETH, A\& BLYTHE, D 2005: Ethics Handbook for Researchers. The Australian and New Zealand Intensive Care Society Clinical Trials Group. Melbourne.

STEWARD, B 2001: Using Nominal Group Technique to Explore Competence in Occupational Therapy and Physiotherapy Students during Firstyear Placements. British Journal of Occupational Therapy. 64(6):298-304.

YOUSUF, MI 2007: Using

Experts' opinion through Delphi Technique. Practical

Assessment. Research and Evaluation 12(4): 1-8. 